PROCEEDINGS OF THE

AMERICAN MATHEMATICAL SOCIETY

Volume 129, Number 8, Pages 2379-2383

S 0002-9939(01)05959-7

Article electronically published on March 15, 2001

\title{
ON THE COMMUTANT OF OPERATORS OF MULTIPLICATION BY UNIVALENT FUNCTIONS
}

\author{
B. KHANI ROBATI AND S. M. VAEZPOUR
}

(Communicated by Joseph A. Ball)

\begin{abstract}
Let $\mathcal{B}$ be a certain Banach space consisting of continuous functions defined on the open unit disk. Let $\phi \in \mathcal{B}$ be a univalent function defined on $\overline{\mathbf{D}}$, and assume that $M_{\phi}$ denotes the operator of multiplication by $\phi$. We characterize the structure of the operator $T$ such that $M_{\phi} T=T M_{\phi}$. We show that $T=M_{\varphi}$ for some function $\varphi$ in $\mathcal{B}$. We also characterize the commutant of $M_{\phi^{2}}$ under certain conditions.
\end{abstract}

\section{IntRoduction}

Let $\mathcal{B}$ be a Banach space consisting of continuous functions defined on the open unit disk $\mathbf{D}$ such that $\mathcal{B}$ satisfies conditions (1)-(6).

(1) $1 \in \mathcal{B}, z \mathcal{B} \subset \mathcal{B}$.

(2) For every $\lambda \in \mathbf{D}$ the evaluation functional at $\lambda$, $e_{\lambda}: \mathcal{B} \rightarrow \mathbf{C}$, given by $f \mapsto f(\lambda)$, is bounded.

(3) $\operatorname{dim} \operatorname{ker}\left(M_{z}-\lambda\right)^{*}=1$ for every $\lambda \in \mathbf{D}$.

(4) If $f \in \mathcal{B}$ and $f$ has an analytic extention to a neighborhood of $\lambda \in \mathbf{D}$, then $\frac{f-f(\lambda)}{z-\lambda} \in \mathcal{B}$. Also for every $\lambda \in \mathbf{D}$ the subspace of $\mathcal{B}$ consisting of those functions in $\mathcal{B}$ that have analytic extention to a neighborhood of $\lambda$ is dense in $\mathcal{B}$.

(5) For every $f \in \mathcal{B}$ the function $\tilde{f}$ defined by $\tilde{f}(\lambda)=f(-\lambda)$ is in $\mathcal{B}$ and $\|\tilde{f}\|=$ $\|f\|$.

(6) If $f \in \mathcal{B}$ and $|f(\lambda)|>c>0$ for every $\lambda \in \mathbf{D}$, then $\frac{1}{f}$ is a multiplier of $\mathcal{B}$.

Throughout this article by a Banach space of continuous functions $\mathcal{B}$ we mean one satisfying the above conditions. A complex valued function $\phi$ defined on $\mathbf{D}$ is called a multiplier of $\mathcal{B}$ if $\phi \mathcal{B} \subset \mathcal{B}$, i.e., $\phi f$ is in $\mathcal{B}$ for every $f$ in $\mathcal{B}$, and the set of all multipliers of $\mathcal{B}$ is denoted by $\mathcal{M}(\mathcal{B})$. As it is shown in [6] each multiplier $\phi$ is bounded on $\mathbf{D}$. Given a multiplier $\phi$, let $M_{\phi}$, defined by $M_{\phi}(f)=\phi f$, denote the operator of multiplication by $\phi$. By the closed graph theorem $M_{\varphi}$ is bounded. The algebra of all bounded operators on $\mathcal{B}$ is denoted by $L(\mathcal{B})$. Let $X \in L(\mathcal{B})$ be a bounded operator on $\mathcal{B}$ and $X M_{z}=M_{z} X$. It is easy to see that $X=M_{\varphi}$ for some function $\varphi \in \mathcal{M}(\mathcal{B})$.

Received by the editors December 16, 1999.

2000 Mathematics Subject Classification. Primary 47B35; Secondary 47 B38.

Key words and phrases. Commutant, multiplication operators, Banach space of analytic functions, univalent function, bounded point evaluation.

Research of the first author was partially supported by a national grant (no. 522).

(C)2001 American Mathematical Society 
Throughout this article $\left\{M_{\varphi}\right\}^{\prime}$ denotes the set of all bounded linear operators $X$ on $\mathcal{B}$ such that $M_{\varphi} X=X M_{\varphi}$, i.e., the commutant of $M_{\varphi}$. Assume $T \in \mathcal{B}^{*}$ and $f \in \mathcal{B}$. We denote the value of $T$ at $f$ by $\langle f, T\rangle$. We define $\hat{M}_{\varphi}: \mathcal{B} \rightarrow \mathcal{B}$ by $\hat{M}_{\varphi}(f)=\varphi \hat{f}$. By the closed graph theorem $\hat{M}_{\varphi}$ is bounded.

In what follows we present some examples of such spaces.

Examples. a) Cole and Gamelin [2] proved that if $\mathcal{A}$ is a T-invariant algebra on a compact set $K$, then for each $\lambda \in K, \operatorname{ran}\left(M_{z}-\lambda\right)$ is dense in kere $\lambda_{\lambda}$. Hence $\operatorname{dim} \operatorname{ker}\left(M_{z}^{*}-\lambda\right)=1$ for every $\lambda \in K$. Also they have shown that every Tinvariant algebra satisfies condition (4). Therefore the algebra of all continuous functions defined on $\mathbf{D}$, i.e., $C(\overline{\mathbf{D}})$, is a Banach space of continuous functions.

b) The disk algebra $A(\mathbf{D})$ which is the algebra of all continuous functions on the closure of disk that are analytic on $\mathbf{D}$.

c) The Bergman space of analytic functions defined on the unit disk $L_{a}^{P}(\mathbf{D})$ for $1 \leq p \leq \infty$.

d) The spaces $D_{\alpha}$ of all functions $f(z)=\sum \hat{f}(n) z^{n}$, holomorphic in $\mathbf{D}$, for which

$$
\|f\|_{\alpha}^{2}=\sum(n+1)^{\alpha}|\hat{f}(n)|^{2}<\infty
$$

for every $\alpha \geq 1$ or $\alpha \leq 0$.

e) The analytic Lipschitz spaces $\mathcal{A}_{\alpha}$ for $0<\alpha<1$, i.e., the space of all analytic functions defined on $\mathbf{D}$ that satisfy a Lipschitz condition of order $\alpha$.

f) The subspace $\mathcal{A}^{\alpha}$ of $\mathcal{A}_{\alpha}$ consisting of functions $f$ in $\mathcal{A}_{\alpha}$ for which

$$
\lim _{z \rightarrow w} \frac{|f(z)-f(w)|}{|z-w|^{\alpha}}=0 .
$$

g) The classical Hardy spaces $H^{p}$ for $1 \leq p \leq \infty$.

Shields and Wallen [6] studied the commutant of the operator $M_{z}$ on the Hilbert spaces of analytic functions. By a slight change in their methods one can obtain the commutant of $M_{z}$ on the Banach spaces of analytic functions. The commutant of a Toeplitz operator on certain Hilbert spaces of functions was studied by many mathematicians. See for example 1, 7], 8. Cuckovic in 3 investigated the commutant of $M_{z^{n}}$ on the Bergman space $L^{2}{ }_{a}(\mathbf{D})$. Seddighi and Vaezpour [5] have shown that under certain conditions on the reproducing kernels of a functional Hilbert spaces every operator $S$ essentially commuting with $M_{z}$ and commuting with $M_{z^{n}}$ for some $n>1$ is a multiplication operator. Also the commutant of $M_{z^{2}}$ on a Banach space of analytic functions and the commutant of $M_{z^{n}}$ on a certain Hilbert space of functions were studied in [4]. In section 2 of this article we characterize the commutant of $M_{\phi}$ for a univalent function $\phi \in \mathcal{M}(\mathcal{B}) \cap A(\mathbf{D})$ on a Banach space of continuous functions and we investigate the commutant of $M_{\phi^{2}}$ under certain conditions.

\section{THE MAIN RESULTS}

Lemma 2.1. If $\phi \in \mathcal{M}(\mathcal{B})$ and $T \in\left\{M_{\phi}\right\}^{\prime}$, then $T^{*}\left(e_{\lambda}\right) \in \operatorname{ker}\left(M_{\phi}-\phi(\lambda)\right)^{*}$ for every $\lambda \in \mathbf{D}$.

Proof. Let $f \in \mathcal{B}$. We have $\left\langle f, M_{\phi}{ }^{*} T^{*}\left(e_{\lambda}\right)\right\rangle=\left\langle f, T^{*} M_{\phi}{ }^{*}\left(e_{\lambda}\right)\right\rangle=\left\langle M_{\phi} T(f), e_{\lambda}\right\rangle=$ $\phi(\lambda) T f(\lambda)=\phi(\lambda)\left\langle T(f), e_{\lambda}\right\rangle=\phi(\lambda)\left\langle f, T^{*}\left(e_{\lambda}\right)\right\rangle=\left\langle f, \phi(\lambda) T^{*}\left(e_{\lambda}\right)\right\rangle ;$ hence $M_{\phi}{ }^{*} T^{*}\left(e_{\lambda}\right)$ $=\phi(\lambda) T^{*}\left(e_{\lambda}\right)$ which implies that $T^{*}\left(e_{\lambda}\right) \in \operatorname{ker}\left(M_{\phi}-\phi(\lambda)\right)^{*}$. 
Theorem 2.2. Let $\phi \in \mathcal{M}(\mathcal{B}) \cap A(\mathbf{D})$ be a univalent map. If $T \in\left\{M_{\phi}\right\}^{\prime}$, then $T=M_{\psi}$ for some function $\psi \in \mathcal{M}(\mathcal{B})$.

Proof. Let $\lambda \in \mathbf{D}$. We show that $\left.\overline{\operatorname{ran}\left(M_{\phi}-\phi(\lambda)\right.}\right)=\operatorname{ker}_{\lambda}$. It is easy to see that $\left.\overline{\operatorname{ran}\left(M_{\phi}-\phi(\lambda)\right.}\right) \subset \operatorname{kere}_{\lambda}$.

To show the converse, let $\phi-\phi(\lambda)=(z-\lambda) g(z)$ by the properties of $\mathcal{B}, g \in \mathcal{B}$. Since $\phi$ is univalent, $g(z) \neq 0$ on $\overline{\mathbf{D}}$ and hence $\frac{1}{g}$ is in $\mathcal{M}(\mathcal{B})$. Now assume that $f \in$ kere $_{\lambda}$ so $f(\lambda)=0$. Since the subspace of $\mathcal{B}$ consisting of functions which are analytic in a neighborhood of $\lambda$ is dense in $\mathcal{B}$, it follows that there is a sequence $\left\{f_{n}\right\}$ of functions in this subspace such that $f_{n}$ tends to $f$. Now assume that $f_{n}-f_{n}(\lambda)=(z-\lambda) g_{n}$ by property $(4)$ of $\mathcal{B}, g_{n} \in \mathcal{B}$. Hence

$$
f_{n}-f_{n}(\lambda)=\frac{\phi-\phi(\lambda)}{g(z)} g_{n}(z)=(\phi-\phi(\lambda)) \frac{g_{n}(z)}{g(z)},
$$

which implies that $\left(f_{n}-f_{n}(\lambda)\right) \in \operatorname{ran}\left(M_{\phi}-\phi(\lambda)\right)$. Since $f_{n}-f_{n}(\lambda)$ tends to $f$ in $\mathcal{B}$, it follows that $f \in \overline{\operatorname{ran}\left(M_{\phi}-\phi(\lambda)\right)}$. Now since $\left(M_{\phi}-\phi(\lambda)\right)^{*}\left(e_{\lambda}\right)=$ $\left(M_{\phi}-\phi(\lambda)\right)^{*} T^{*}\left(e_{\lambda}\right)=0$ and $\operatorname{dim} \operatorname{ker}\left(M_{\phi}-\phi(\lambda)\right)^{*}=1$, we conclude that $T^{*}\left(e_{\lambda}\right)=$ $\psi(\lambda) e_{\lambda}$ for some constant $\psi(\lambda)$. Therefore, we have

$$
T(f)(\lambda)=\left\langle T(f), e_{\lambda}\right\rangle=\left\langle f, T^{*}\left(e_{\lambda}\right)\right\rangle=\psi(\lambda)\left\langle f, e_{\lambda}\right\rangle=\psi(\lambda) f(\lambda) .
$$

Hence $T(f)=\psi f$ for every $f \in \mathcal{B}$ and the proof is complete.

In the remainder of this section we investigate the commutant of $M_{\phi^{2}}$ for some univalent function $\phi$.

Corollary 2.3. If $\phi \in \mathcal{M}(\mathcal{B}) \cap A(\mathbf{D})$ is a univalent map such that $\phi(\overline{\mathbf{D}})$ has no distinct points which are symmetric with respect to the origin, then $\left\{M_{\phi^{2}}\right\}^{\prime}=\left\{M_{\psi}\right.$ : $\psi \in \mathcal{M}(\mathcal{B})\}$. In particular if $|\lambda|>1$, then $\left\{M_{(z-\lambda)^{2}}\right\}^{\prime}=\left\{M_{\psi}: \psi \in \mathcal{M}(\mathcal{B})\right\}$.

Remark. In the proofs of Lemma 2.1 and Theorem 2.2 we did not use property (5) of Banach space $\mathcal{B}$. Also $\mathbf{D}$ can be replaced by every bounded open set $G$.

Lemma 2.4. Let $\phi$ be a univalent odd function in $\mathcal{M}(\mathcal{B}) \cap A(\mathbf{D}), S \in L(\mathcal{B})$ and $S M_{\phi}=-M_{\phi} S$. Then there exists $\psi \in \mathcal{M}(\mathcal{B})$ such that $S=\hat{M}_{\psi}$.

Proof. Since $T^{*} M_{\phi}^{*}=-M_{\phi}^{*} T^{*}$ by a similar argument asin the proof of Lemma 2.1, we have $M_{\phi}^{*} T^{*}\left(e_{\lambda}\right)=-\phi(\lambda) T^{*}\left(e_{\lambda}\right)$; hence $\left(M_{\phi}+\phi(\lambda)\right)^{*}\left(T^{*}\left(e_{\lambda}\right)\right)=0$ which yields $T^{*}\left(e_{\lambda}\right) \in \operatorname{ker}\left(M_{\phi}+\phi(\lambda)\right)^{*}$. By the proof of Theorem 2.2, $e_{-\lambda} \operatorname{spans} \operatorname{ker}\left(M_{\phi}+\phi(\lambda)\right)^{*}$ so $T^{*}\left(e_{\lambda}\right)=\psi(\lambda) e_{-\lambda}$ for some constant $\psi(\lambda)$. Now

$$
\left\langle T(f), e_{\lambda}\right\rangle=\left\langle f, T^{*}\left(e_{\lambda}\right)\right\rangle=\psi(\lambda)\left\langle f, e_{-\lambda}\right\rangle=\psi(\lambda) f(-\lambda)
$$

which implies that $T=\hat{M}_{\psi}$. Also $T(\hat{f})=\psi f$; hence $\psi \in \mathcal{M}(\mathcal{B})$.

Theorem 2.5. Let $\phi \in \mathcal{M}(\mathcal{B}) \cap A(\mathbf{D})$ be an odd univalent map. Let $S \in\left\{M_{\phi^{2}}\right\}^{\prime}$ and $S M_{\phi}-M_{\phi} S$ be a compact operator. Then there is some $\Psi \in \mathcal{M}(\mathcal{B})$ such that $S=M_{\Psi}$.

Proof. We have $\left(S M_{\phi}-M_{\phi} S\right) M_{\phi}=\left(S M_{\phi^{2}}-M_{\phi} S M_{\phi}\right)=M_{\phi^{2}} S-M_{\phi} S M_{\phi}=$ $-M_{\phi}\left(S M_{\phi}-M_{\phi} S\right)$. Hence, by Lemma 2.4 there exists some $\psi \in \mathcal{B}$ such that $S M_{\phi}-M_{\phi} S=\hat{M}_{\psi}$. Now we show that $M_{\psi}$ is compact. Let the operator $T$ be 
defined by $T(f)=\hat{f}$; it is obvious that $T$ is continuous. Now we have $M_{\psi}(f)=$ $\hat{M}_{\psi} T(f)$ for every $f \in \mathcal{B}$ and so $M_{\psi}$ is compact and by the Fredholm alternative theorem $\psi=0$. This implies that $M_{\phi} S=S M_{\phi}$; hence $S \in\left\{M_{\phi}\right\}^{\prime}$ and we conclude that $S=M_{\Psi}$ for some $\Psi \in \mathcal{M}(\mathcal{B})$.

Theorem 2.6. Let $\phi \in \mathcal{M}(\mathcal{B}) \cap A(\mathbf{D})$ be an odd univalent map. Suppose $T$ is an operator in $\left\{M_{\phi^{2}}\right\}^{\prime}$ and let $T M_{\phi}+M_{\phi} T$ be a compact operator. Then there exists $a \Psi \in \mathcal{M}(\mathcal{B})$ such that $T=\hat{M}_{\Psi}$.

Proof. We have $\left(T M_{\phi}+M_{\phi} T\right) M_{\phi}=M_{\phi}\left(T M_{\phi}+M_{\phi} T\right)$; hence by Theorem 2.2, there is a function $\psi \in \mathcal{M}(\mathcal{B})$ such that $T M_{\phi}+M_{\phi} T=M_{\psi}$. Since $T M_{\phi}+M_{\phi} T$ is compact, we have $\psi=0$, so $M_{\phi} T=-M_{\phi} T$. Now, by Lemma 2.4, there is $\Psi \in \mathcal{M}(\mathcal{B})$ such that $T=\hat{M}_{\Psi}$.

Theorem 2.7. Let $\phi \in \mathcal{M}(\mathcal{B}) \cap A(\mathbf{D})$ be a univalent map of $\mathbf{D}$ onto $\mathbf{D}$ such that $f \circ \phi$ and $f \circ \phi^{-1}$ are in $\mathcal{B}$ for every $f \in \mathcal{B}$. Let $S \in\left\{M_{\phi^{2}}\right\}^{\prime}$. If polynomials are dense in $\mathcal{B}$, then $S(f)=\Phi f+\psi \frac{f+\left(\widehat{f \circ \Phi^{-1}}\right) \circ \Phi}{2 \phi}$, where $S(1)=\Phi$ and $\left(S M_{\phi}-M_{\phi} S\right)(1)=\psi$.

Proof. We define $T: \mathcal{B} \rightarrow \mathcal{B}$ by $T(f)=f \circ \phi^{-1}$. Clearly $T \in L(\mathcal{B})$ with inverse $T^{-1}(f)=f \circ \phi$. Since $M_{z} T=T M_{\phi}$, by induction we have $M_{z^{n}} T=T M_{\phi^{n}}$ for every positive integer $n$. Since $S M_{\phi^{2}}=M_{\phi^{2}} S$, it follows that $S T^{-1} M_{z^{2}} T=T^{-1} M_{z^{2}} T S$ and so $T S T^{-1} \in\left\{M_{z^{2}}\right\}^{\prime}$. Now by a similar argument as in the proof of [4, Theorem 2.6] we have

$$
T S T^{-1}(f)=T S T^{-1}(1)(f)+\left(T S T^{-1} M_{z}-M_{z} T S T^{-1}\right)(1)\left(\frac{f+\hat{f}}{2 z}\right) .
$$

If $S(1)=\Phi$ and $\left(S M_{\phi}-M_{\phi} S\right)(1)=\psi$, then $T S T^{-1}(1)=\Phi \circ \phi^{-1}$. Since

$$
T\left(S M_{\phi}-M_{\phi} S\right) T^{-1}=T S T^{-1} M_{z}-M_{z} T S T^{-1},
$$

it follows that $\left(T S T^{-1} M_{z}-M_{z} T S T^{-1}\right)(1)=\psi \circ \phi^{-1}$. Hence

$$
S(f)=T^{-1} T S T^{-1} T(f)=\Phi f+\psi \frac{f+\left(\widehat{f \circ \Phi^{-1}}\right) \circ \Phi}{2 \phi} .
$$

Remark. If $\phi$ in Theorem 2.7 is an odd function, then $S(f)=\Phi f+\psi \frac{f+\hat{f}}{2 \phi}$.

\section{REFERENCES}

[1] S. Axler and Z. Cuckovic, Commuting Toeplitz operators with harmonic symbols, Integral Equation Operator Theory, 14(1991), 1-12. MR 92f:47018

[2] B.J. Cole and T.W. Gamelin, Tight uniform algebras and algebras of analytic functions, J. Funct. Anal. 46(1982),158-220. MR 83h:46065

[3] Z. Cuckovic, Commutant of Toeplitz operators on the Bergman spaces, Pacific. J. Math., 162(1994), 277- 285. MR 94j:47041

[4] B. Khani Robati, On the commutant of certain multiplication operators on spaces of analytic functions, Rendiconti del circolo matematico di Palermo, to appear.

[5] K. Seddighi and S. M. Vaezpour, Commutant of certain multiplication operator on Hilbert spaces of analytic functions, Studia Math., 133(2)(1999), 121-130. MR 2000i:47062

[6] A. L. Shields and L. J. Wallen, The commutants of certain Hilbert space operators, Indiana Univ. Math. J., 20 (1971), 777- 788. MR 44:4558 
[7] J. E. Thomson, Intersections of commutants of analytic Toeplitz operators, Proc. Amer. Soc., 52(1975), 305-310. MR 53:3765

[8] J. E. Thomason, The commutant of certain analytic Toeplitz operators, Proc. Amer. Soc., 54(1976), 165-169. MR 52:8993

Department of Mathematics, Shiraz University, Shiraz 71454, Iran

E-mail address: Khani@math.susc.ac.ir

Department of Mathematics, Yazd University, Yazd, Iran 